EPJ Web of Conferences 16, 04006 (2011)

DOI: $10.1051 /$ epjconf/20111604006

(C) Owned by the authors, published by EDP Sciences, 2011

\title{
Physical parameters of a sample of $M$ dwarfs from high-resolution near-infrared spectra
}

\author{
C. del Burgo ${ }^{1,2, a}$, R. Deshpande ${ }^{3}$, E.L. Martín ${ }^{4,3}$, M.R. Zapatero Osorio ${ }^{4}$, \\ S. Witte ${ }^{5}$, Ch. Helling ${ }^{6}$ and P.H. Hauschildt ${ }^{5}$ \\ 1 UNINOVA-CA3, Campus da Caparica, Quinta da Torre, Monte de Caparica, \\ 2825-149 Caparica, Portugal \\ 2 School of Cosmic Physics, Dublin Institute of Advanced Studies, Dublin 2, Ireland \\ 3 University of Central Florida, Department of Physics, Orlando, FL 32816-2385, USA \\ ${ }^{4}$ CAB (CSIC-INTA), Ctra. Ajalvir km 4, 28850 Torrejón de Ardoz, Madrid, Spain \\ ${ }^{5}$ Hamburger Sternwarte, Gojenbergsweg 112, 21029 Hamburg, Germany \\ 6 SUPA, School of Physics and Astronomy, University of St. Andrews, North Haugh, \\ St. Andrews KY16 9SS, UK
}

\begin{abstract}
We show preliminary results about the effective temperature, surface gravity, and projected rotational velocity of eleven $\mathrm{M}$ dwarfs from the comparison of high-resolution near-infrared spectra with resolving power of $\sim 20,000$ and atmosphere synthetic models. Our results are in good agreement with those found in the literature. We find that the haze of observed absorption features (mostly water vapor) is relatively well modeled by the synthetic spectra.
\end{abstract}

\section{INTRODUCTION}

This study is part of an effort to characterize a sample of nearby late M-dwarfs, with spectral types between M5 and M9.5, from a program of radial velocity (RV). The final objective is to discover habitable rocky planets around them. This is the main science driver of a few trailblazer projects aimed at developing high-resolution near-infrared spectrographs to measure RVs with unprecedented precision (see, e.g., [1, 2], Martín et al., these Proc.).

In the spectral type range of interest here, most objects are expected to be very low-mass cool stars, although some of them could be young brown dwarfs. In fact, in the well-characterized Pleiades cluster, the transition between stars and brown dwarfs takes place at a spectral type of M6.5 (see [3]). It has been estimated that the substellar-mass boundary is located at M9 for an age of $1 \mathrm{Gyr}$ (see [4]). Determination of the surface gravity in late-M dwarfs can yield constraints on their age and mass, and hence on their stellar or substellar status. An early attempt to carry out this type of analysis was made by [5] for the very low-mass binary GJ569 Bab.

The comparison of high-resolution spectroscopy with synthetic models of the atmospheres of stars provides a powerful tool to study their properties (chemical composition, effective temperature, rotational velocity and surface gravity). We have recently applied the technique of matching theoretical spectra to high-resolution data to derive the physical properties of a sample of nine T dwarfs (see [6]). Here we present a preliminary study of 11 out of the 40 late-M dwarfs of our sample observed with the instrument NIRSPEC (see [7]) on the Keck II telescope. We include Van Biesbroeck 10 (VB 10) in our analysis. A detailed study of the RV curve for VB10 is reported in these proceedings (Deshpande et al.) and in [8].

aemail: cburgo@uninova.pt

This is an Open Access article distributed under the terms of the Creative Commons Attribution-Noncommercial License 3.0, which permits unrestricted use, distribution, and reproduction in any noncommercial medium, provided the original work is properly cited. 
Table 1. Physical properties of the M dwarfs.

\begin{tabular}{|l|l|l|l|l|l|l|l|l|l|l|}
\hline Object & SpT & $\begin{array}{c}\mathbf{v}_{\text {rot }} \text { sini } \\
\mathbf{k m ~ s}^{-\mathbf{1}}\end{array}$ & $\begin{array}{c}\text { Prev. } \\
\mathbf{k m ~ s}^{-\mathbf{1}}\end{array}$ & $\mathbf{R e f .}$ & $\begin{array}{c}\mathbf{T}_{\text {eff }} \\
\mathbf{K}\end{array}$ & $\begin{array}{c}\text { Prev. } \\
\mathbf{K}\end{array}$ & $\begin{array}{l}\text { Ref. } \\
\text { Logg } \\
\text { [cgs] }\end{array}$ & $\begin{array}{c}\text { Prev. } \\
{[\mathbf{c g s}]}\end{array}$ & Ref. \\
\hline RXJ2208 & M5.0e & $17 \pm 5$ & & & $3260 \pm 200$ & 3170 & {$[13]$} & $5.3 \pm 0.3$ & & \\
\hline GJ1002 & M5.5 & $5 \pm 1$ & $\leq 3$ & {$[14]$} & $3130 \pm 160$ & 3150 & {$[14]$} & $5.3 \pm 0.4$ & & \\
\hline GJ905 & M5.5 & $4 \pm 1$ & $\leq 3$ & {$[14]$} & $3240 \pm 140$ & 3240 & {$[14]$} & $5.3 \pm 0.4$ & & \\
\hline LP860-41 & M6.0 & $17 \pm 7$ & & & $2867 \pm 218$ & 2620 & {$[15]$} & $4.9 \pm 0.7$ & & \\
\hline LHS1363 & M6.5 & $8 \pm 5$ & & & $2988 \pm 35$ & & & $5.3 \pm 0.4$ & & \\
\hline GJ406 & M6.5e & $4 \pm 1$ & 3 & {$[14]$} & $2975 \pm 46$ & 3150 & {$[14]$} & $5.3 \pm 0.4$ & 5.5 & {$[16]$} \\
& & & & & & 2900 & {$[16]$} & & & \\
\hline LHS1937 & M7.0 & $11 \pm 6$ & & & $2800 \pm 262$ & & & $5.1 \pm 0.5$ & & \\
\hline LHS3003 & M7.0 & $4 \pm 2$ & 6.0 & {$[14]$} & $2838 \pm 192$ & 2620 & {$[14]$} & $5.2 \pm 0.4$ & 4.5 & {$[16]$} \\
\hline VB10 & M8.0 & $10 \pm 1$ & $\sim 10$ & {$[17]$} & $2670 \pm 240$ & 2700 & {$[18]$} & $4.9 \pm 0.5$ & & \\
\hline LP349-25 & M8.0 & $63 \pm 3$ & & & $2680 \pm 270$ & & & $4.9 \pm 0.7$ & & \\
\hline 2MJ1733 & M9.5 & $26 \pm 4$ & & & $2500 \pm 400$ & & & $4.8 \pm 0.8$ & & \\
\hline
\end{tabular}

\section{OBSERVATIONS, DATA REDUCTION, MODELS AND DATA PREPARATION}

\subsection{Observations}

The observations were performed with the high-resolution near-infrared spectrograph NIRSPEC, which is installed at the KECK II telescope in Hawaii. The instrumental setup was selected to observe ten useful echelle orders to cover part of the $\mathrm{J}$ band from 1.148 to $1.346 \mu \mathrm{m}$, a nominal dispersion ranging from 0.164 (blue wavelengths) to $0.191 \AA \mathrm{pix}^{-1}$ (red wavelengths), and a final resolution element of $0.55-0.70 \AA$ at $1.2485 \mu \mathrm{m}$, i.e., about the central wavelength, corresponding to a resolving power $\mathrm{R}$ of about 20,000 (see [9]). For consistency we applied the data processing described in [9].

\subsection{Description of the atmosphere models}

We have used two distinct atmosphere models, depending on the effective temperature $\left(\mathrm{T}_{\text {eff }}\right)$ range of interest. The general-purpose stellar atmosphere PHOENIX code (see [10]) was used for $\mathrm{T}_{\text {eff }}$ above $3000 \mathrm{~K}$. In particular, we used the version 16 that includes a number of improvements compared to previous versions, such as a complete new equation of state for ions, molecules and condensation (ACES; Barman et al., in prep.), updated opacity databases, and improved line profiles for atomic lines. For $\mathrm{T}_{\text {eff }}$ below $3000 \mathrm{~K}$, we use the Drift-PHOENIX code, which is a merger of the PHOENIX code and the dust model Drift (see [11]). The dust grains are composites and yield improved opacities in contrast to the grains in earlier models, and the use of a non-phase-equilibrium chemistry avoids an overestimated condensation/evaporation.

\subsection{Data preparation}

The synthetic models were transformed for a proper comparison with the observations as explained in del Burgo et al. (2009). First, models were transformed to take into account the projected rotational velocity ( $\mathrm{v}_{\text {rot }} \operatorname{sini}$ ) of the objects using the formalism of Gray (see [12]). Then, they were convolved with a Gaussian that mimics the instrumental profile along the dispersion axis. The resulting spectra were finally rebinned to the same resolution of the observations and normalized over the wavelength range corresponding to order 59. 
Research, Science and Technology of Brown Dwarfs and Exoplanets

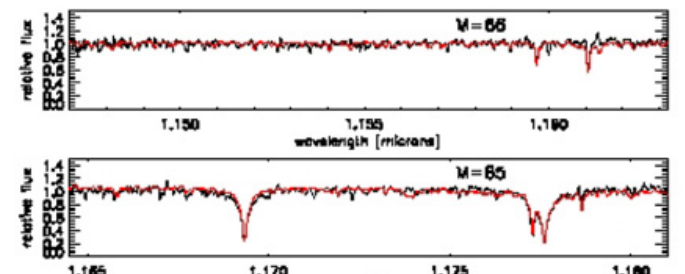

1.165

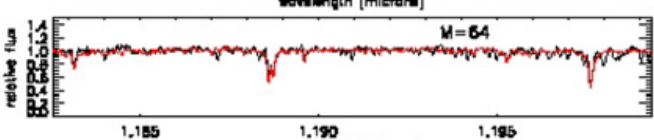

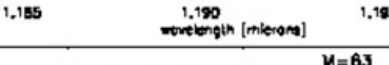
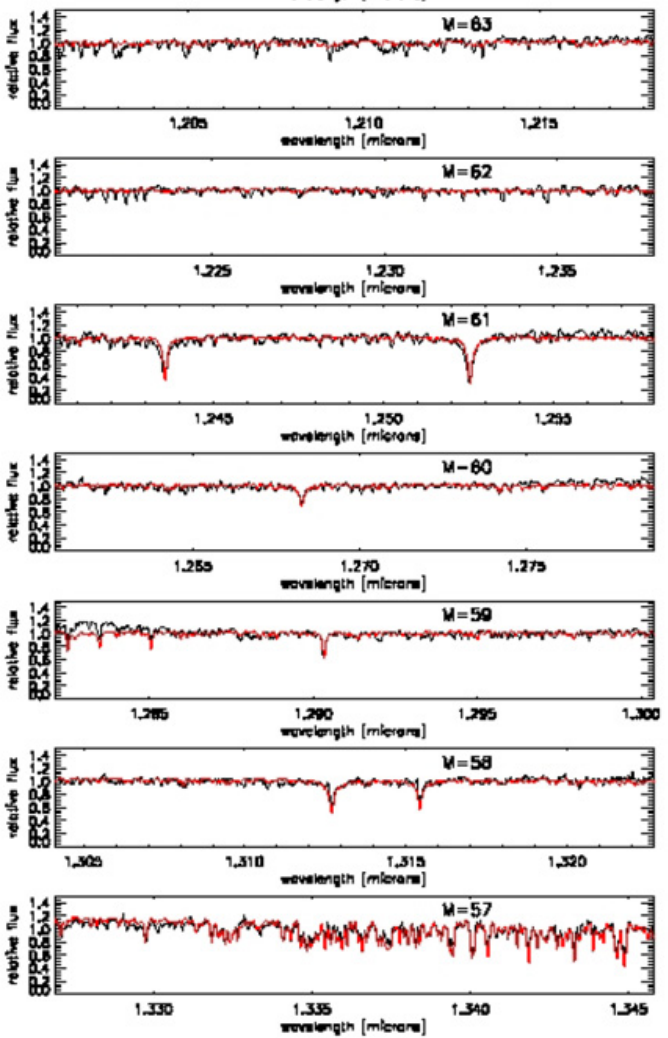
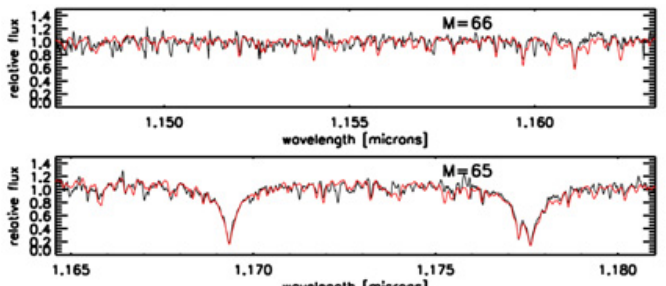

$1.165 \quad 1,170$ movelength [microns] 1.175
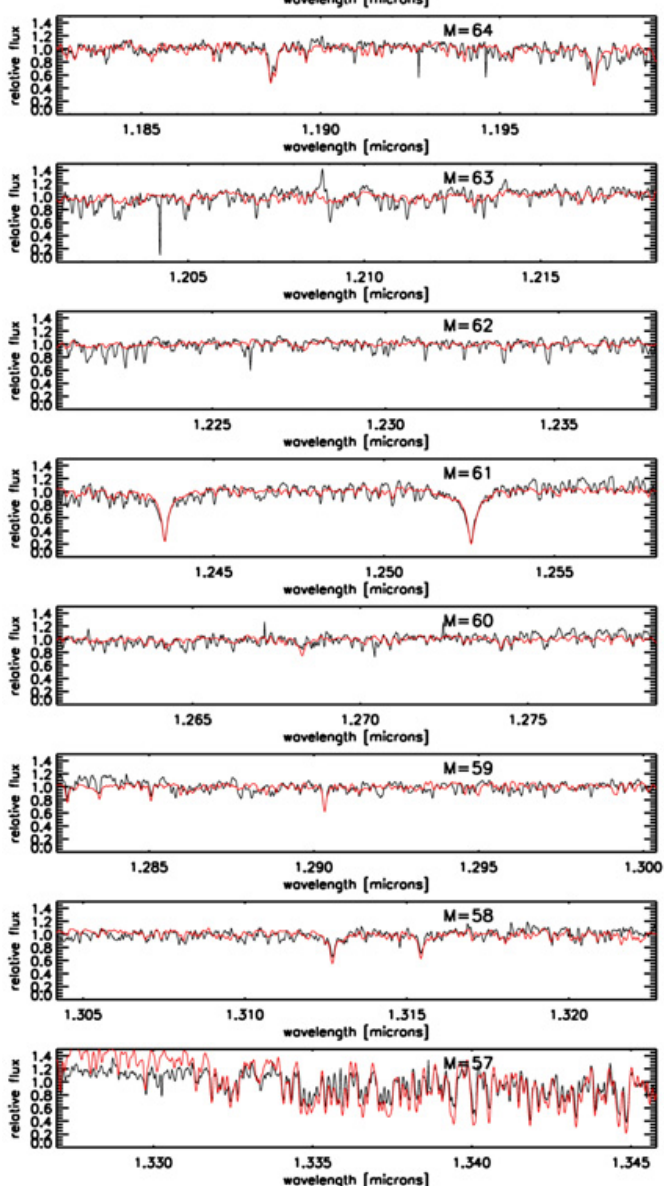

Figure 1. Spectra observed (black) and modelled (red) corresponding to GJ 406 (left) and VB10 (right).

A grid of synthetic models with solar metallicity, $\mathrm{v}_{\mathrm{rot}} \operatorname{sini}$ in the range from 0 to $75 \mathrm{~km} \mathrm{~s}^{-1}$ with steps of 1 or $2 \mathrm{~km} \mathrm{~s}^{-1}, \mathrm{~T}_{\text {eff }}$ between 1000 and $4000 \mathrm{~K}$ with steps of $100 \mathrm{~K}$, and $\operatorname{logg}$ between 3.5 and 5.5 (cgs) with steps of 0.5 was generated. Observed spectra were moved to vacuum wavelengths for a proper comparison with the theoretical models. This was done from a cross-correlation analysis with each individual synthetic spectrum that allow us to determine RVs.

\section{ANALYSIS AND RESULTS}

In order to constrain the number of possible solutions for the properties of each object provided by our large set of models, we determine the minimum of the root-mean-square $R M S$. See [6] for a detailed 
description of the method. Table 1 shows the results for the eleven $M$ dwarfs, where the spectral type $(\mathrm{SpT})$ is indicated together with $\mathrm{v}_{\mathrm{rot}} \mathrm{sini}, \mathrm{T}_{\text {eff }}, \log$ and the values found in the literature.

We note that the synthetic models nicely reproduce the haze of absorption features (see Fig. 1 for two examples of our sample) with abundant water vapor, although the strength of some of the faintest features is not well matched. Some of the mismatching is due to the line modeling, but also to problems in the flat-fielding (e.g., order 57 in VB10) and the subtraction of some telluric lines (e.g., order 62). We are currently investigating alternative techniques to improve the telluric subtraction (see Rodler et al. in these Proceedings).

\section{References}

[1] E. L. Martín, E. Guenther, D. Barrado y Navascués, et al., Astronomische Nachrichten, 326, 1015 (2005)

[2] L. W. Ramsey, J. Barnes, S. L. Redman, et al., PASP, 120, 887 (2008)

[3] E. L. Martín, R. Rebolo, M. R. Zapatero Osorio ApJ, 469, 706 (1996)

[4] A. Magazzu, R. Rebolo, M. R. Zapatero Osorio, et al., ApJ, 497, L47 (1998)

[5] M. R. Zapatero Osorio, B. F. Lane, Y. Pavlenko, et al., ApJ, 615, 958 (2004)

[6] C. del Burgo, E. L. Martín, M. R. Zapatero Osorio, P. H. Hauschildt, A\&A, 501, 1059 (2009)

[7] I. S. McLean, E. E. Becklin, O. Bendiksen, et al., SPIE, 3354, 566 (1998)

[8] M. R. Zapatero Osorio, E. L. Martín, C. del Burgo, et al., A\&A Letters, 505, 5 (2009)

[9] M. R. Zapatero Osorio, E. L. Martín, H. Bouy, et al., ApJ, 647, 1406 (2006)

[10] P. H. Hauschildt, E. Baron, JCoAM, 109, 41 (1999)

[11] Ch. Helling, P. Woitke, W. -F. Thi, 2008, A\&A, 485, 547 (2008)

[12] D. F. Gray, "The Observations and Analysis of Stellar Photospheres", Cambridge University Press, 2nd. ed (1992)

[13] F.-J. Zickgraf, J. Krautter, S. Reffert, et al., A\&A, 433, 151 (2005)

[14] A. Reiners, G. Basri, ApJ, 656, 1121 (2007)

[15] N. Phan-Bao, F. Crifo, X. Delfosse, et al., A\&A, 401, 959 (2003)

[16] B. Fuhrmeister, J. H. M. M. Schmitt, P. H. Hauschildt, A\&A, 439, 1137 (2005)

[17] H. R. A. Jones, Y. Pavlenko, S. Viti, et al., MNRAS, 358, 105 (2005)

[18] A. Schweitzer, P. H. Hauschildt, F. Allard, G. Basri, MNRAS, 283, 821 (1996) 